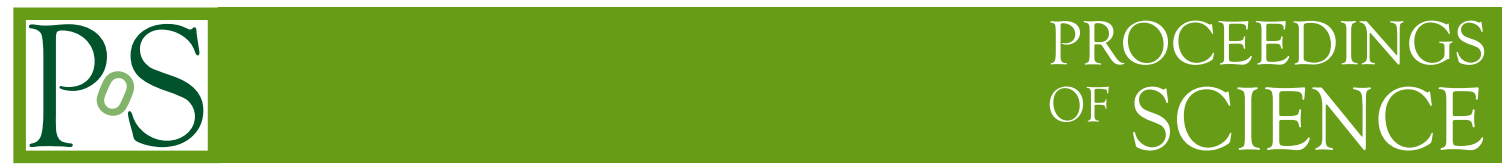

\title{
Higgs boson measurements in third generation decay channels (excluding ttH) with ATLAS and CMS
}

\author{
Jiggins, Stephen ${ }^{a, *}$ on behalf of the ATLAS and CMS Collaborations \\ ${ }^{a}$ Albert-Ludwigs Universität Freiburg, \\ Hermann-Herder-Straße 3a, 79104 Freiburg im Breisgau, Germany \\ E-mail: stephen.jiggins@cern.ch
}

\begin{abstract}
The coupling of the Higgs $(H)$ boson to $3^{\text {rd }}$ generation fermions serves as a powerful tool for precision measurements of the Standard Model, but also as a probe for Beyond the Standard Model physics. The following article will review the latest Higgs to $3^{\text {rd }}$ generation fermion decay measurements from the ATLAS and CMS experiments, excluding direct $t t H$ measurements, using LHC proton-proton collision data at $\sqrt{s}=13 \mathrm{TeV}$. Particular emphasis is placed on the latest measurements of $H \rightarrow b \bar{b}$ via associated vector boson production $(V H)$ from ATLAS, an inclusive search for $H \rightarrow b \bar{b}$ via gluon fusion ( $g g F$ ) plus jet production from CMS, and a CP-invariance test using $H \rightarrow \tau \tau$ via vector-boson fusion production (VBF).
\end{abstract}

The Eighth Annual Conference on Large Hadron Collider Physics-LHCP2020

25-30 May, 2020

online

${ }^{*}$ Speaker 


\section{Introduction}

The discovery of the Higgs boson [1-4] in 2012 by the ATLAS and CMS Collaborations [5, 6] using proton-proton ( $p p$ ) collision data produced by the Large Hadron Collider [7] (LHC), was a fundamentally important turning point for the Standard Model (SM). The Higgs-to-boson couplings were determined via the well established boson decay modes $\left(H \rightarrow W W^{*} / Z Z^{*}\right)$ using data collected in 2011 and 2012 (Run 1), and have since entered an era of precision measurements [8-11].

The direct coupling of the Higgs boson to the fermionic sector on the other hand was only established via the observation of $H \rightarrow \tau \tau$ following the Run 1 ATLAS and CMS combination [12]. The dominant $H \rightarrow b \bar{b}$ decay mode was not observed until 2018 [13, 14] when the addition of the 2015-2017 Run 2 ATLAS and CMS datasets was made available. Following these milestones, further measurement of Higgs to $3^{\text {rd }}$ generation fermion couplings offers a wide range of novel opportunities, from limit setting of invisible/undetected decays originating from beyond SM (BSM) physics [9, 15], to probes of CP-violation from $H \rightarrow V V$ anomalous couplings [16, 17].

This article will therefore summarise a total of four Higgs to $3^{\text {rd }}$ generation fermion analyses recently published by the ATLAS and CMS Collaborations. Two additional analyses pertaining to the measurement of the $H \rightarrow \tau \tau$ decay mode using a total integrated luminosity of $77.4 \mathrm{fb}^{-1}$, and a BSM probe of constraints on anomalous $H V V$ couplings from production of Higgs bosons decaying to $\tau$ lepton pairs using an integrated luminosity of $35.9 \mathrm{fb}^{-1}$, published via the CMS Collaboration are available in Refs. [17, 18], respectively. These however will not be covered in this article as they have been published and summarised in previous proceedings.

\section{Measurements of $H \rightarrow \tau \tau$ via vector-boson fusion}

A recent result from ATLAS using $H \rightarrow \tau \tau$ via VBF production as a test of CP-invariance was published [19]. Utilising $p p$ collision data at a centre-of-mass energy of $\sqrt{s}=13 \mathrm{TeV}$ collected by the ATLAS detector with an integrated luminosity of $36.1 \mathrm{fb}^{-1}$, a model-independent effective Lagrangian density with CP-odd operators of mass dimension six involving the Higgs field and electroweak gauge fields parameterised according to Ref. [20] is tested as a possible extension to the SM. The effective Lagrangian ( $\left.\mathcal{L}_{\text {eff }}\right)$ written in the mass basis of the Higgs boson $(H)$, photon $(A)$, and weak gauge bosons $\left(W^{ \pm} / Z^{0}\right)$ yields a squared VBF matrix element of the form:

$$
|\mathcal{M}|=\mathcal{M}_{\mathrm{SM}}^{2}+\tilde{d} \cdot 2 \mathcal{R} e\left(\mathcal{M}_{\mathrm{SM}}^{*} \mathcal{M}_{\mathrm{CP}-\text { odd }}\right)+\tilde{d}^{2} \cdot \mathcal{M}_{\mathrm{CP}-\text { odd }}^{2},
$$

where $\mathcal{M}_{\mathrm{SM}}$ represents the VBF SM matrix element, and $\mathcal{M}_{\mathrm{CP}-\text { odd }}$ is the dimension six CP-odd anomalous coupling matrix element. CP-violation in VBF Higgs production is therefore described by a single CP-violating strength parameter $\tilde{d}$ [21]. A genuine CP-odd Optimal Observable (OO) [21] that has a (non-)vanishing expectation value under the (CP-violating) SM hypothesis, is utilised as the analysis fit discriminant:

$$
\langle O O\rangle=\frac{2 \operatorname{Re}\left(\mathcal{M}_{\mathrm{SM}}^{*} \mathcal{M}_{\mathrm{CP}-\mathrm{odd}}\right)}{\left|\mathcal{M}_{\mathrm{SM}}\right|^{2}} .
$$

Figure 1a summarises the $\triangle$ NLL likelihood scan of the data, and various expectations, as a function of the CP-violating strength parameter $\tilde{d}$, whilst Table $1 \mathrm{~b}$ summarises the mean values of 


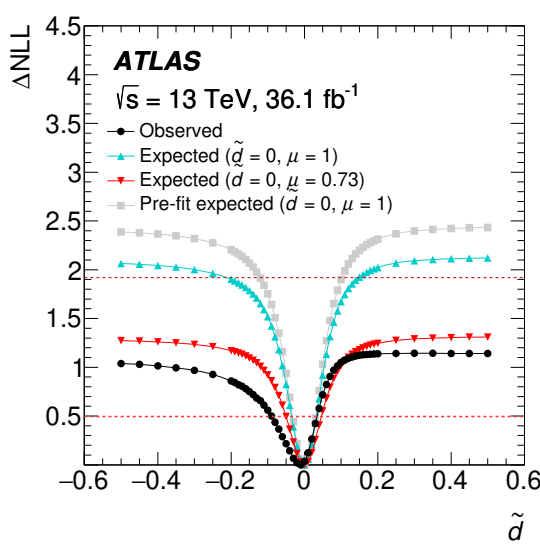

(a)

\begin{tabular}{lc}
\hline Channel & 〈Optimal Observable $\rangle$ \\
\hline$\tau_{\text {lep }} \tau_{\text {lep }}$ SF & $-0.54 \pm 0.72$ \\
$\tau_{\text {lep }} \tau_{\text {lep }}$ DF & $0.71 \pm 0.81$ \\
$\tau_{\text {lep }} \tau_{\text {had }}$ & $0.74 \pm 0.78$ \\
$\tau_{\text {had }} \tau_{\text {had }}$ & $-1.13 \pm 0.65$ \\
\hline Combined & $-0.19 \pm 0.37$ \\
\hline
\end{tabular}

(b)

Figure 1: The observed $\triangle N L L$ curve as a function of the CP-violating strength parameter $\tilde{d}$ (a), and observed mean values of $\langle O O\rangle$ (b). For comparison, expected $\triangle$ NLL curves are also shown, generated using pseudo-data sets [19].

the $O O$ for the four $\tau$ decay signatures (di-leptonic, leptonic+hadronic, and di-hadronic). The two demonstrate agreement of the data with no additional anomalous forms of CP-violation, thereby setting limits on anomalous CP-violating $H V V$ couplings of $-0.11<\tilde{d}<0.05$ at a $68 \%$ confidence level.

\section{Measurements of $H \rightarrow b \bar{b}$ via associated vector-boson production}

Following the discovery of the $H \rightarrow b \bar{b}$ decay mode in 2018 [13, 14], two new $H \rightarrow b \bar{b}$ measurements of $V\left(=W^{ \pm} / Z^{0}\right) H$ production have since been published by the ATLAS Collaboration. The first referred to as resolved $V H b b$ [22], reconstructs fully leptonic $W / Z$-boson decays, whilst the Higgs-boson candidate is reconstructed via requiring exactly two $b$-tagged anti- $k_{\mathrm{t}} \mathrm{R}=0.4$ jets. The second, referred to as boosted $V H b b$ [23], adopts the same reconstruction strategy for the leptonically decaying $W / Z$-boson, but the Higgs-boson is reconstructed using an anti- $k_{\mathrm{t}} \mathrm{R}=1.0$ jet (large-R) with two matched $b$-tagged variable radius anti- $k_{\mathrm{t}}$ trackjets to the large-R jet. The former targets a region of phase space where the transverse momentum of the Higgs candidate $\left(p_{\mathrm{T}}^{\mathrm{H}}\right)$ exceeds approximately $60 \mathrm{GeV}$, whilst the latter targets $p_{\mathrm{T}}^{\mathrm{H}} \gtrsim 250 \mathrm{GeV}$.

Both analyses use the full Run 2 ATLAS $p p$ dataset of $139 \mathrm{fb}^{-1}$, quoting results on the ratio of the observed vs SM predicted $V H$ production cross-section times $H \rightarrow b \bar{b}$ branching $\left(\mu_{V H}^{b \bar{b}}\right)$, Simplified Template Cross-Section (STXS) measurements [24], and Warsaw basis Standard Model low energy Effective Field Theory (EFT) interpretations [25]. For the resolved $V H b b$ analysis $\mu_{V H}^{b b}=1.02_{-0.11}^{+0.12}$ (stat. $)_{-0.13}^{+0.14}$ (syst.) is observed, rejecting the background-only hypothesis with a significance of 6.7 standard deviations. Separate results for $W H$ and $Z H$ are also quoted as shown in Figure 2a, with data excesses above the background-only hypothesis of 4.0 and 5.3 standard deviations in significance, respectively.

For the boosted $V H b b$ analysis $\mu_{V H}^{b b}=0.72_{-0.28}^{+0.29}$ (stat. $)_{-0.22}^{+0.26}$ (syst.) is observed with a data excess above the background-only hypothesis of 2.1 standard deviations. Figure $2 \mathrm{~b}$ summarises the $\mu_{V H}^{b b}$ 


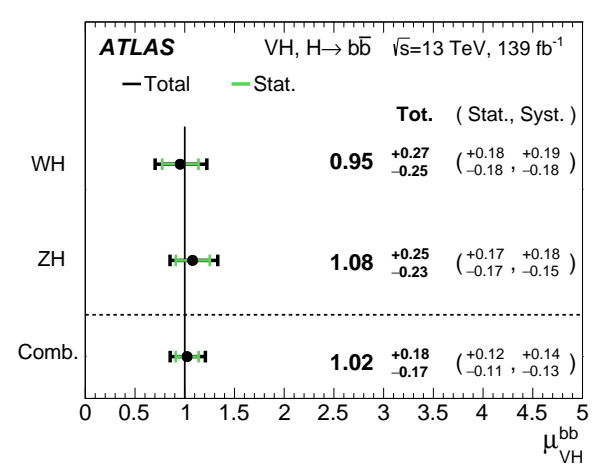

(a) $V H b b$ resolved $\mu_{V H}^{b b}$ best-fit values

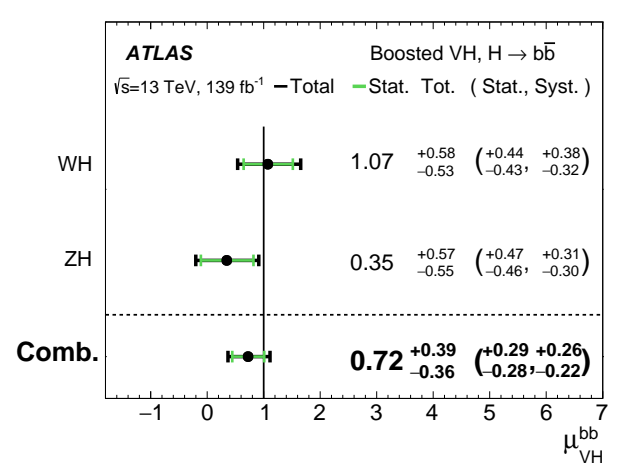

(b) $V H b b$ boosted $\mu_{V H}^{b b}$ best-fit values

Figure 2: Signal strength of $V H$ production cross-section times $H \rightarrow b \bar{b}$ branching ratio, $\mu_{V H}^{b b}$, best-fit values, and corresponding errors, for the (a) resolved [22], and (b) boosted [23] analyses.

values for $W H, Z H$, and the combined $V H$ result.

\section{Measurements of $H \rightarrow b \bar{b}$ via gluon fusion plus jets}

Gluon fusion production of $H \rightarrow b \bar{b}$ in association with additional QCD initial state radiation, referred to as $g g F+$ jet, provides an alternative indirect approach to measuring the top Yukawa coupling, in addition to disentangling long-/short-range contributions to $g g F$ production [26].

The recent CMS measurement of $g g F+$ jet production in the $H \rightarrow b \bar{b}$ decay mode [27] probes the $p_{\mathrm{T}}^{\mathrm{H}} \approx 450 \mathrm{GeV}$ region of phase space through the reconstruction of the Higgs candidate via a single anti- $k_{\mathrm{t}} \mathrm{R}=0.8$ jet trimmed/groomed using the Soft-drop algorithm $(z=0.1, \beta=0)$ [28]. Identification of the $H \rightarrow b \bar{b}$ decay is made via a deep-neural network double b-tagger [29] with a $33 \%$ efficiency for the $H \rightarrow b \bar{b}$ signal and a $1 \%$ miss-tag rate for QCD multijet background. The additional QCD radiation is identified via the reconstruction of a single anti- $k_{\mathrm{t}} \mathrm{R}=0.4$ radius jet.

An inclusive jet $p_{\mathrm{T}}$ signal strength parameter of $\mu_{H}=3.7 \pm 1.2$ (stat. $)_{-0.7}^{+0.6}(\text { syst. })_{-0.5}^{+0.8}$ (theo.) is measured, corresponding to a 2.5(1.9) $\sigma$ excess above the background-only (SM signal) hypothesis. In addition, an unfolded differential measurement of gluon fusion production cross-section times $H \rightarrow b \bar{b}$ branching ratio $\left(\sigma_{g g F}^{b b}\right)$ is made using a maximum likelihood unfolding technique like that derived in Section 5 of Ref. [30]. The unfolded differential cross-section is compared to theoretical predictions from Ref. [31] and Powheg+HJ+MiNLO [32], as shown by Figure 3, where inclusively the data exceed the SM prediction with a $1.9 \sigma$ significance, or a local $2.6 \sigma$ significant excess for the third $p_{\mathrm{T}}$ bin.

\section{Conclusion}

A review of the most recent results from the ATLAS and CMS Collaborations pertaining to the measurement of $H \rightarrow b \bar{b}$ and $H \rightarrow \tau \tau$ decay modes has been presented. In the area of Standard Model precision measurements two analyses from the ATLAS Collaboration targeting the associated vector boson production mode $(\mathrm{VH})$ provide updated measurements of the production cross-section, STXS, and tests of EFT extensions in the Warsaw basis with the full Run 2 (2015-2018) dataset. 


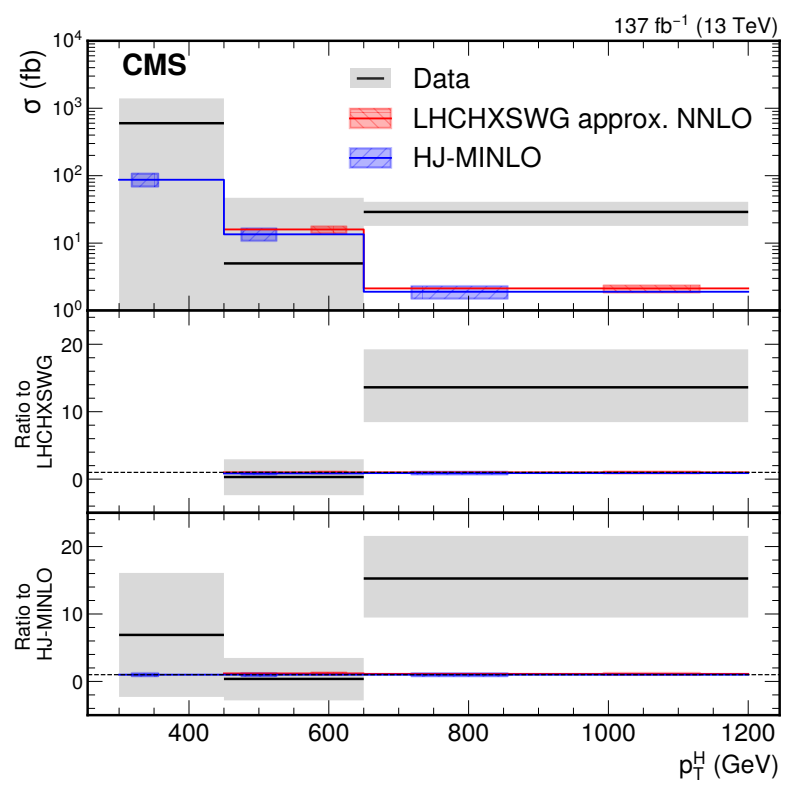

Figure 3: Unfolded differential distribution as a function of the large-R jet $p_{\mathrm{T}}$ for $g g \rightarrow H+$ jet production, compared to LHC cross-section working NNLO prediction from Ref. [31], and to PowHEG+HJ+MiNLO [32] MC generator cross-section prediction [27].

From the CMS Collaboration, a measurement of the fiducial differential gluon fusion cross-section as a function of $p_{\mathrm{T}}^{\mathrm{H}}$ was presented. Addressing the area of BSM physics, a probe of CP-violation from $H \rightarrow V V$ anomalous couplings in the Higgs and electroweak sectors using $H \rightarrow \tau \tau$ via VBF production from the ATLAS Collaboration was presented. 


\section{References}

[1] P. W. Higgs, Broken Symmetries and the Masses of Gauge Bosons, Phys. Rev. Lett. 13 (1964) 508-509.

[2] F. Englert and R. Brout, Broken Symmetry and the Mass of Gauge Vector Mesons, Phys. Rev. Lett. 13 (1964) 321-323.

[3] P. W. Higgs, Broken symmetries, massless particles and gauge fields, Physics Letters 12 (1964) 132 133.

[4] G. S. Guralnik, C. R. Hagen, and T. W. B. Kibble, Global Conservation Laws and Massless Particles, Phys. Rev. Lett. 13 (1964) 585-587.

[5] ATLAS Collaboration, Observation of a new particle in the search for the Standard Model Higgs boson with the ATLAS detector at the LHC, Physics Letters B 716 (2012) 1 - 29.

[6] CMS Collaboration, Observation of a new boson at a mass of $125 \mathrm{GeV}$ with the CMS experiment at the LHC, Physics Letters B 716 (2012) $30-61$.

[7] A. Breskin and R. Voss, The CERN Large Hadron Collider: Accelerator and Experiments. CERN, Geneva, 2009. https: //cds . cern. ch/record/1244506.

[8] ATLAS and CMS Collaborations, Combined Measurement of the Higgs Boson Mass in pp Collisions at $\sqrt{s}=7$ and $8 \mathrm{TeV}$ with the ATLAS and CMS Experiments, Physical Review Letters 114 (2015) 191803 .

[9] ATLAS Collaboration, Combined measurements of Higgs boson production and decay using up to $80 \mathrm{fb}^{-1}$ of proton-proton collision data at $\sqrt{\mathrm{s}}=13 \mathrm{TeV}$ collected with the ATLAS experiment, Phys. Rev. D 101 (2020) 012002, arXiv: 1909.02845 [hep-ex].

[10] CMS Collaboration, Measurement of inclusive and differential Higgs boson production cross sections in the diphoton decay channel in proton-proton collisions at $\sqrt{s}=13 \mathrm{TeV}$, Journal of High Energy Physics 2019 (2019) 183.

[11] CMS Collaboration, Measurements of properties of the Higgs boson decaying to a W boson pair in pp collisions at $\sqrt{s}=13$ TeV, Physics Letters B 791 (2019) 96-129.

[12] ATLAS and CMS Collaborations, Measurements of the Higgs boson production and decay rates and constraints on its couplings from a combined ATLAS and CMS analysis of the LHC pp collision data at $\sqrt{s}=7$ and $8 \mathrm{TeV}$, JHEP 08 (2016) 045, arXiv: 1606.02266 [hep-ex].

[13] ATLAS Collaboration, Observation of $H \rightarrow b \bar{b}$ decays and VH production with the ATLAS detector, Physics Letters B 786 (2018) 59 - 86.

[14] CMS Collaboration, Observation of Higgs Boson Decay to Bottom Quarks, Phys. Rev. Lett. 121 (2018) 121801.

[15] CMS Collaboration, Combined Higgs boson production and decay measurements with up to $137 \mathrm{fb}-1$ of proton-proton collision data at $\sqrt{s}=13 \mathrm{TeV}$, CMS-PAS-HIG-19-005, 2020, https://cds. cern.ch/record/2706103.

[16] ATLAS Collaboration, Test of CP invariance in vector-boson fusion production of the Higgs boson using the Optimal Observable method in the ditau decay channel with the ATLAS detector, The European Physical Journal C 76 (2016) 658, arXiv: 1602.04516 [hep-ex]. 
[17] CMS Collaboration, Constraints on anomalous HVV couplings from the production of Higgs bosons decaying to $\tau$ lepton pairs, Phys. Rev. D 100 (2019) 112002, arXiv: 1903.06973 [hep-ex].

[18] CMS Collaboration, Measurement of Higgs boson production and decay to the $\tau \tau$ final state, CMS-PAS-HIG-18-032, 2019, https: //cds . cern. ch/record/2668685.

[19] ATLAS Collaboration, Test of CP invariance in vector-boson fusion production of the Higgs boson in the $H \rightarrow \tau \tau$ channel in proton-proton collisions at $\sqrt{s}=13 \mathrm{TeV}$ with the ATLAS detector, Phys. Lett. B 805 (2020) 135426, arXiv:2002.05315 [hep-ex].

[20] V. Hankele, G. Klamke, D. Zeppenfeld, and T. Figy, Anomalous Higgs boson couplings in vector boson fusion at the CERN LHC, Phys. Rev. D 74 (2006) 095001, arXiv: hep-ph/0609075 [hep-ph].

[21] M. Davier, L. Duflot, F. Le Diberder, and A. Rougé, The optimal method for the measurement of tau polarization, Physics Letters B 306 (1993) 411-417.

[22] ATLAS Collaboration, Measurements of $W H$ and $Z H$ production in the $H \rightarrow b \bar{b}$ decay channel in pp collisions at $13 \mathrm{TeV}$ with the ATLAS detector, arXiv: 2007.02873 [hep-ex].

[23] ATLAS Collaboration, Measurement of the associated production of a Higgs boson decaying to $b$ quarks with a vector boson at high transverse momentum in pp collisions at $\sqrt{s}=13 \mathrm{TeV}$ with the ATLAS detector, ATLAS-CONF-2020-007, 2020, https : //cds . cern. ch/record/2715063.

[24] LHC Higgs Cross Section Working Group Collaboration, D. de Florian et al., Handbook of LHC Higgs Cross Sections: 4. Deciphering the Nature of the Higgs Sector, arXiv: 1610.07922 [hep-ph].

[25] B. Grzadkowski, M. Iskrzynski, M. Misiak, and J. Rosiek, Dimension-Six Terms in the Standard Model Lagrangian, JHEP 10 (2010) 085, arXiv: 1008. 4884 [hep-ph].

[26] C. Grojean, E. Salvioni, M. Schlaffer, and A. Weiler, Very boosted Higgs in gluon fusion, JHEP 05 (2014) 022, arXiv: 1312.3317 [hep-ph].

[27] CMS Collaboration, Inclusive search for highly boosted Higgs bosons decaying to bottom quark-antiquark pairs in proton-proton collisions at $\sqrt{s}=13 \mathrm{TeV}$, arXiv:2006.13251 [hep-ex].

[28] A. J. Larkoski, S. Marzani, G. Soyez, and J. Thaler, Soft Drop, JHEP 05 (2014) 146, arXiv: 1402.2657 [hep-ph].

[29] CMS Collaboration, Identification of double-b quark jets in boosted event topologies, CMS-PAS-BTV-15-002, 2016, https: //cds . cern. ch/record/2195743.

[30] CMS Collaboration, Measurement and interpretation of differential cross sections for Higgs boson production at $\sqrt{s}=13 \mathrm{TeV}$, Physics Letters B 792 (2019) 369-396, arXiv: 1812.06504 [hep-ex].

[31] K. Becker et al., Precise predictions for boosted Higgs production, arXiv:2005.07762 [hep-ph].

[32] K. Hamilton, P. Nason, C. Oleari, and G. Zanderighi, Merging $H / W / Z+O$ and 1 jet at NLO with no merging scale: a path to parton shower + NNLO matching, JHEP 05 (2013) 082, arXiv: 1212.4504 [hep-ph]. 